\title{
Longstanding liver cysts and obstructive jaundice
}

\author{
E Roberts, I A Finnie
}

A 79-year-old woman from a semi-rural area of North Wales was referred because of intermittent jaundice (bilirubin $80 \mu \mathrm{mol} / \mathrm{l}$ ), deranged liver function (albumin $20 \mathrm{~g} / \mathrm{l}$ ) malaise, anorexia and weight loss. Results of computed tomography (CT), endoscopic retrograde cholangiopancreatography (ERCP), and plain abdominal X-ray are shown in figures 1-3, respectively. Plain abdominal X-ray was known to be abnormal in 1968.

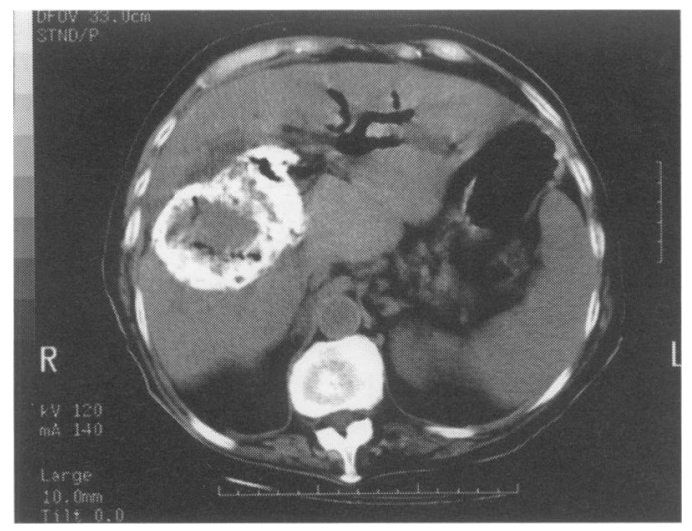

Figure 1 Unenhanced CT scan of abdomen

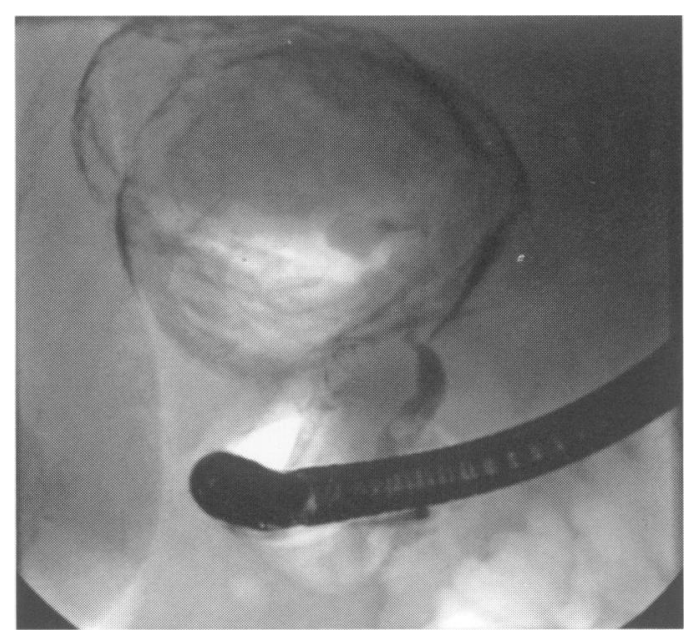

Figure 2 Early film from the ERCP

\section{Questions}

1 Describe the abnormalities seen in figures 1 and 2 and state the probable diagnosis.

2 What procedure has been done (figure 3 )?

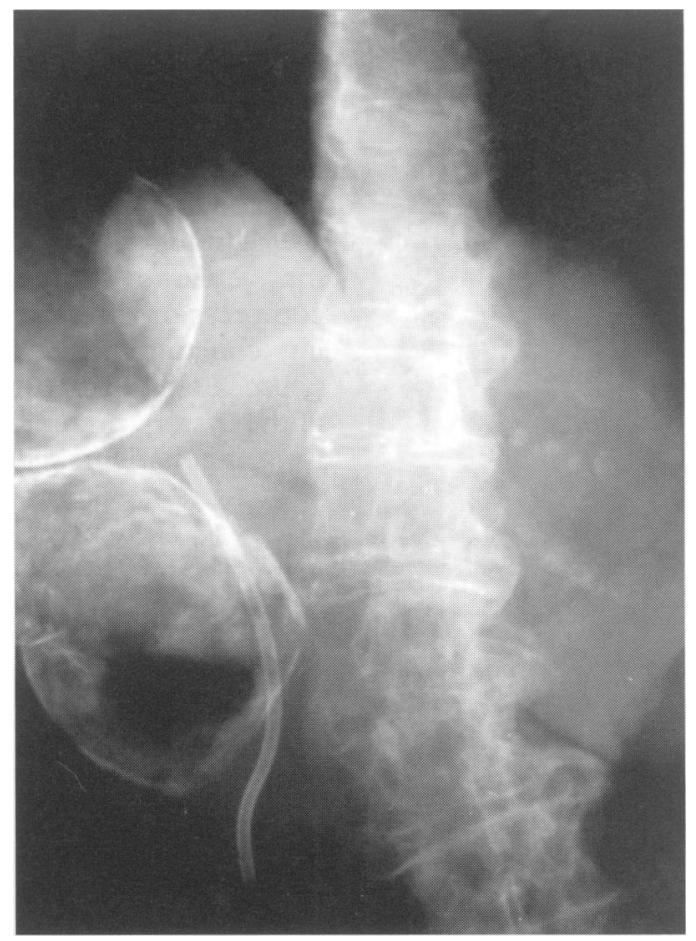

Figure 3 Plain abdominal X-ray \section{Hospital, Rhyl, \\ Denbighshire \\ E Roberts \\ I A Finnie}

ment of

Dr Finnie

Accepted 10 March 1998 


\section{Answers}

\section{QUESTION 1}

The CT scan (figure 1) shows two large calcified cysts in the right lobe of the liver. Air is present within the cysts and in the left hepatic duct. The ERCP (figure 2) shows a tight, smooth stricture of the common bile duct approximately $2 \mathrm{~cm}$ long adjacent to the lower cyst. There is early filling of the cyst.

The diagnosis is hydatid (Echinococcus granulosus) disease of the liver.

QUESTION 2

The abdominal X-ray (figure 3) shows that an endoprosthesis has been placed across the stricture.

\section{Discussion}

Hydatid disease is caused by infection with $E$ granulosus, a canine tapeworm. ${ }^{12}$ The disease may affect multiple organs, but most commonly the liver $(60 \%)$. When the liver is affected, the most common area is inferiorly in the right lobe. The cysts may be asymptomatic, may enlarge causing abdominal pain, may compress or rupture into the biliary tree causing cholangiitis, or rupture into the peritoneum. Standard treatment is surgical removal of the cyst(s), preferably complete and intact. ${ }^{1-3}$ In some patients chemotherapy, particularly with albendazole, may be effective ${ }^{23}$; some authors have reported excellent results from ultrasound-guided cyst aspiration combined with albendazole. ${ }^{4}$ These non-surgical approaches were unlikely to succeed in our patient with large calcified cysts.

Although biliary obstruction and stricturing have been well documented in hydatid disease, they have often been attributed to the treatment. ${ }^{5}$ The ERCP appearances in our case suggest that the biliary stricture occurred as a result of cyst enlargement. Following placement of the prosthesis the patient's appetite improved, the jaundice disappeared, and appetite returned to normal. Serum albumin was normal 2 months later.

Biliary communication occurs in a substantial proportion of cases of hydatid disease in some series, and endoscopic palliation should be considered in patients who may have biliary strictures, but who are unfit for surgery.

\section{Final diagnosis}

Hydatid (Echinococcus granulosus) disease of the liver.

Keywords: echinococcosis; jaundice; tapeworm; endoscopic retrograde cholangiopancreatography; stents; hyatid disease

We thank Dr Jane Wood for referring the patient.
1 Fenton-Lee D, Morris DL. The management of hydatid disease of the liver: Part 1. Trop Doct 1996;26:173-6.

2 Fenton-Lee D, Morris DL. The management of hydatid Fenton-Lee D, Morris DL. The management of
disease of the liver: Part 2. Trop Doct 1997;27:87-8.

3 disease of the liver: Part 2. Trop Doct 1997;27:87-8.

3 Morris DL. Echinococcus of the liver. Gut 1994;35:1517-8. compared with surgery for hepatic hydatid cysts. $N$ Engl $\mathcal{F}$ Med 1997;337:881-7.
5 Castellano G, Moreno-Sanchez D, Gutierrez J, MorenoGonzalez E, Colina F, Solis-Herruzo JA. Caustic sclerosing cholangitis. Report of four cases and a cumulative review of the literature. Hepatogastroenterology 1994;41:458-70. 